\title{
Effect of Bile Salts on the Interfacial Dilational Rheology of Lecithin in the Lipid Digestion Process
}

\author{
Aicha Mekkaoui ${ }^{1,2}$, Yang Liu', Pingping Zhang ${ }^{1}$, Sana Ullah ${ }^{1,2}$, Ce Wang ${ }^{1,2 *}$ and \\ Baocai $\mathrm{Xu}^{1,2 *}$ \\ ${ }^{1}$ School of Light Industry, Beijing Technology and Business University, Beijing 100048, CHINA \\ ${ }^{2}$ Beijing Advanced Innovation Center for Food Nutrition and Human Health, Beijing Technology and Business University, Beijing 100048, CHINA
}

\begin{abstract}
The effects of bile salts on the emulsifier adsorption layer play a crucial role in lipid digestion. The current study selected sodium cholate $(\mathrm{NaCh})$ and lecithin as model compounds for bile salts and food emulsifiers, respectively. The interface dilational rheological and emulsification properties of $\mathrm{NaCh}$ and lecithin were carried out. The results showed that the $\mathrm{NaCh}$ molecules could quickly diffuse from the bulk to interface, which broke the tightly-arranged interfacial layer of lecithin and enhanced the viscoelasticity of interfacial film. As a result, the interfacial adsorption layer, which was originally dominated by the slow relaxation processes within the interface, was transformed into one controlled by the fast molecular diffusion exchange. This accelerated the exchange of materials between the bulk and interface, thereby creating suitable conditions for the interfacial adsorption of lipases, which promoted the digestion process. These results provided a mechanism for the promotion of lipid digestion by bile salts from the perspective of interfacial viscoelasticity and relaxation processes. A deeper understanding of the interfacial behavior of bile salts with emulsifiers would provide a basis for the rational design of interfacial layer for modulating lipid digestion.
\end{abstract}

Key words: bile salt, lecithin, emulsion, lipid digestion, interfacial dilational rheology

\section{Introduction}

During the last decade, the occurrence of obesity and other long-term chronic diseases, such as cardiovascular disease, diabetes and certain cancers, is continuously increasing worldwide, which is mainly due to the changing lifestyles and patterns of food consumption. High-fat diet is a major contributor, causing the imbalance of energy intake and appearance of unhealthy conditions ${ }^{1}$. The regulation of bioavailability of dietary fats by controlling lipid digestion is believed as an effective approach to prevent the increasing obesity and other related diseases ${ }^{2}$. Currently, increasing studies are focusing on the reduction of rate and degree of lipids digestion using functional foods, for effective reduction of lipid absorption and promotion of satiety ${ }^{3-6)}$.

In brief, the lipid digestion mainly occurs in gastrointestinal tract. When the digested food is transported into small intestine, the lipids are mixed with pancreatic juice, containing bicarbonate of soda, bile salts, phospholipids, pancreatic lipase, and co-lipase. As a result of interfacial activity, the bile salts facilitate the emulsification of lipids by adsorbing to the surfaces of droplets and contribute to the binding of enzymes to lipid substrates ${ }^{7)}$. For the lipid emulsions stabilized with emulsifiers before entering small intestine, the interfacial films prevent lipases from coming into close proximity to lipids. In this case, the bile salts compete and displace the pre-adsorbed amphiphiles at the interface, which makes it possible for lipases to bind to the surface of lipid droplets. Then, the hydrolysis of triacylglycerols takes places under the catalytic action of pancreatic lipase/co-lipase ${ }^{8)}$. After that, the products of lipid digestion, including diacylglycerol/monoacylglycerol(DAG/ MAG) and free fatty acid (FFA), and the components dissolved in lipids, such as oil-soluble vitamins, phospholipids, and cholesterol, are removed from the interface by the bile salts and form mixed micelles or vesicles, which then pass through the mucous layer that covers the epithelium walls and finally reach to enterocyte cells ${ }^{9,10)}$. It can be seen that bile salts play a significant role in the interfacial process of lipid digestion.

As a class of amphiphilic molecules secreted by human body, bile salts are derived from cholesterol and have a steroidal core structure ${ }^{11)}$. Unlike classical amphiphiles that

\footnotetext{
*Correspondence to: Ce Wang, School of Light Industry, Beijing Technology and Business University, Beijing 100048, CHINA; Baocai Xu, School of Light Industry, Beijing Technology and Business University, Beijing 100048, CHINA

E-mail: wangce@btbu.edu.cn (CW); xubaoc@ btbu.edu.cn (BX).

Accepted March 31, 2021 (received for review March 3, 2021)

Journal of Oleo Science ISSN 1345-8957 print / ISSN 1347-3352 online

http://www.jstage.jst.go.jp/browse/jos/ http://mc.manusriptcentral.com/jjocs
} 
consist of hydrophilic head-groups and hydrophobic tails, bile salts exhibit planar polarity. Their hydrophobic surface lies on the convex side of the rigid steroid ring structure with angular methyl groups at positions C-18 and C-19, while the hydrophilic surface lies on the concave side with one, two, or three hydroxyl groups and a carboxyl group conjugated with taurine, glycine or other amino acids ${ }^{7}$. Due to this unique structure, they are less effective in terms of lowering the interfacial tension(IFT) than many other surfactants, but more effective in terms of competing with other amphiphiles at interface ${ }^{12,13)}$. This has been proved by detection of changes in zeta-potential, IFT, or interfacial dilational modulus before and after the addition of bile salts ${ }^{14-20)}$. The designing of an appropriate interface adsorption film, inhibiting the competitive adsorption of bile salts, is a well-accepted feasible approach for the regulation of lipid digestion ${ }^{21-23)}$. However, it is still unclear how the bile salts displace emulsifier at the oil-water interface, because the intermolecular interactions and relaxation processes during the replacement cannot be well explained simply by monitoring the changes of zeta-potential, IFT, or interfacial dilational modulus. But, this is important for the rational design of emulsification system for the regulation of lipid digestion. A better understanding of the interfacial processes responsible for the displacement of emulsifiers by bile salts is still needed ${ }^{24)}$.

According to the literature, the measurement of the dilational rheological properties of interface can provide a credible means for the detection of its viscoelasticity and relaxation processes, reflecting the interaction of molecules at the interface ${ }^{25-31)}$. The use of this technique in physiological interfacial issues has now been receiving increasing attention $^{32)}$. The microscopic interfacial behaviors of amphiphiles can be inferred by detecting the dependence of interfacial viscoelasticity on the concentration of amphiphiles, interfacial oscillating frequency, and interfacial pressure. Therefore, it was used to study the interfacial dilational rheological behaviors of emulsifiers in the presence of bile salts in the present work.

Lecithin is one of the most widely used food emulsifiers, containing two non-polar fatty acids, which are esterified into the backbone of glycerol with a polar phosphate headgroup attached to a hydrophilic residue, such as inositol, choline, serine or ethanolamine ${ }^{33-35)}$. As zwitterionic surfactants, lecithin can effectively lower oil-water IFT and stabilize oil-in-water emulsions ${ }^{36)}$. In the current study, soybean lecithin was used as a representative of exogenous food emulsifiers. On the other hand, despite the varieties, all the bile salts behave qualitatively in a similar manner. Herein, sodium cholate (NaCh) was used as an endogenous bile salt. The interactions between lecithin and bile salts have attracted increasing attention because of their ability to form mixed micelles that facilitate the digestion of lipids in intestine ${ }^{37-39)}$. However, studies on the interfacial inter- action between these two amphiphiles are rarely reported.

The emulsification and interfacial behaviors of lecithin, $\mathrm{NaCh}$, and their mixture were studied in this work. The interfacial behaviors were assessed by the IFT and interfacial dilational rheology at the oil-aqueous interface using oscillating drop method. The emulsification behaviors were estimated by the distribution of droplet size and destabilization index. Finally, the in vitro lipid digestion of lecithinstabilized emulsion was carried out in the absence and presence of NaCh, respectively. Remarkably, the presence of NaCh greatly altered the interfacial viscoelasticity of lecithin, which provided a mechanism of the promotion of lipid digestion by bile salts from the perspective of interfacial dilatation rheology.

\section{Materials and Methods}

\subsection{Materials}

$\mathrm{NaCh}$ hydrate was obtained from Beijing Budweiser Technology CO., Ltd. Soybean lecithin was obtained from Tokyo Chemical Industry CO., Ltd. The molecular structures of NaCh and Soybean lecithin are shown in Scheme 1. Medium-chain triacylglycerols (MCT) were obtained from Shanghai Yuanye Bio-Technology Co., Ltd. Tris (hydroxymethyl) methyl aminomethane was obtained from Shanghai Macklin Bio-Technology Co., Ltd. Hydrochloric acid ( $\mathrm{HCl})$ and $\mathrm{NaOH}$ were obtained from Sinopharm Chemical Reagent Co., Ltd. Sodium chloride $(\mathrm{NaCl})$ and calcium chloride $\left(\mathrm{CaCl}_{2}\right)$ were obtained from Beijing J\&K Scientific Ltd. Lipase was obtained from Shanghai Yuanye Bio-Technology Co., Ltd. Ultrapure water with a resistivity of 18.2 $\mathrm{M} \Omega . c m$ was used for the preparations of solutions. $n$-Decane, with a purity of $99.5 \%$, was obtained from Beijing Budweiser Technology CO., Ltd. All the other chemicals used in this study were of analytical grade.

\subsection{Interfacial tension and dilatational rheological mea- surements}

The measurements of IFT and interfacial dilational rheology were taken using an oscillating drop tensiometer ODG-20 (DataPhysics Instruments GmbH, Germany). This instrument mainly composed of a computer-controlled syringe with a needle for droplet formation, quartz cuvette,
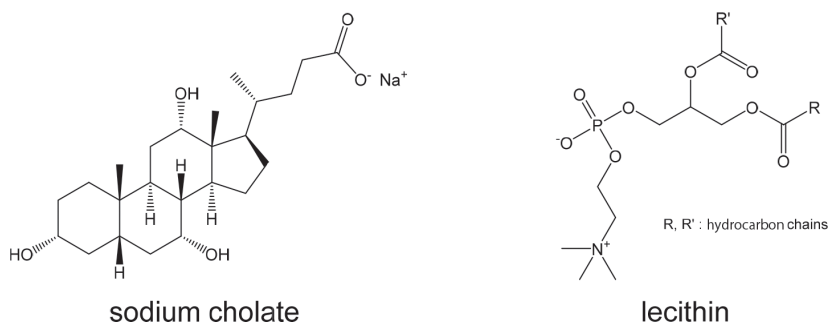

Scheme 1 Molecular structures of NaCh and lecithin. 


\section{Bile Salts Accelerate the Diffusion Exchange of Materials between the Bulk and Interface}

CCD camera, light source, and a frame grabber. The interface was created by injecting an oil droplet from a reversed stainless steel needle into the aqueous phase in cuvette. A sinusoidal periodic compression and expansion of the interface area $(A)$ was performed at a selected amplitude $(\Delta A$ / $A, 10 \%)$ at a frequency of $0.1 \mathrm{~Hz}$. The image of the droplet was taken with the CCD camera. $\operatorname{IFT}(\gamma)$ was calculated by fitting the Laplace equation to the shape of the droplet. As primarily defined by Gibbs, the dilational modulus $(\varepsilon)$ can be expressed as given in Eq. (1).

$$
\varepsilon=\frac{\mathrm{d} \gamma}{\mathrm{d} \ln A}
$$

The interfacial dilational modulus can also be expressed as a complex number, which contains a real portion (storage modulus), representing the elastic energy kept in the interface, and an imaginary portion(loss modulus)resulted from interfacial relaxation processes, which match to the elasticity $\left(\varepsilon_{\mathrm{d}}\right)$ and viscosity $\left(\varepsilon_{\eta}=\omega \eta \mathrm{d}\right)$, respectively, as given in Eq. (2) ${ }^{40)}$.

$$
\varepsilon=\varepsilon_{\mathrm{d}}+i \omega \eta \mathrm{d}
$$

When the adsorption of amphiphiles at interface reached equilibrium, the interfacial oscillations at different frequencies $(0.005 \sim 0.1 \mathrm{~Hz})$ were carried out to study the interfacial viscoelasticity.

All the measurements were performed at $30^{\circ} \mathrm{C}$. The complex composition of MCT makes it difficult to analyze the interface behavior of amphiphilic molecules. Therefore, $n$-decane was selected as simulation oil in this experiment. Lecithin was dissolved in the $n$-decane as oil phase, while $\mathrm{NaCh}$ was dissolved in water as aqueous phase. It should be noted that, for the lecithin oil solution-NaCh aqueous solution systems, the concentration of $\mathrm{NaCh}$ in aqueous phase and that of lecithin in oil phase were equal, and the concentrations in the results and discussion part were equal to the sums of the concentration of $\mathrm{NaCh}$ in the aqueous phase and the concentration of lecithin in the oil phase.

\subsection{Preparation of emulsion}

$\mathrm{NaCh}$ was dissolved in water at different concentrations (0.1 wt \% , $0.5 \mathrm{wt} \%, 1.0 \mathrm{wt} \%$, and $1.5 \mathrm{wt} \%$ ) by stirring gently until complete dissolution. The $\mathrm{pH}$ of aqueous solutions was adjusted to 7 , according to the condition of small intestine. The oil phase was prepared by dispersing lecithin in MCT and stirring until complete dissolution. The oil and aqueous phases were mixed with a volume ratio of $2: 8(\mathrm{O} /$ W) using a high-shear mixer (15-081, Ningbo Scientz Biotechnology Co., Ltd) at a speed of $8000 \mathrm{rpm}$ for $3 \mathrm{~min}$ to form coarse emulsion. The emulsion was then homogenized using a Panda PLUS processor (GEA Niro Soavi Corporation, Italy) at the operational pressure of $800 \mathrm{MPa}$ for five times.

\subsection{Determination of droplet size}

The droplet size of emulsions was determined using a dynamic light scattering instrument (Zetasizer Nano ZS, Malvern, U.K.). In order to avoid multiple scattering effects, the samples were diluted 100 times with ultrapure water prior to taking measurements. The droplet sizes were calculated from the average of 5 readings taken using the same sample. All the measurements were carried out at least in duplicate with three prepared samples.

\subsection{Stability of emulsion}

Multiple light scattering measurements were taken for the evaluation of the stability of emulsion using a commercial apparatus, Turbiscan (Formulation, L'Union, France). The freshly prepared emulsion samples were placed in cylindrical quartz tubes and the measurements were taken at $30^{\circ} \mathrm{C}$ for $24 \mathrm{~h}$. The dispersion of emulsion was analyzed using two detectors $\left(0^{\circ}\right.$ from the incident beam-the transmitted light, and $135^{\circ}$ from the incident beam-the backscattered light). The turbiscan stability index(TSI), the sum of all the variations in the intensity of backscattering light at different locations of the test tube, could reflect the stability of emulsions. This could be calculated using Eq. (3) as following: ${ }^{41)}$

$$
T S I=\sum_{j}\left|\operatorname{scan}_{\text {ref }}\left(h_{j}\right)-\operatorname{scan}_{i}\left(h_{j}\right)\right|
$$

where $\operatorname{scan}_{\text {ref }}$ and $\operatorname{scan}_{i}$ are the initial backscattering value and backscattering at a given time point, and $h_{j}$ is a given height in the measuring cylindrical glass tube. In general, the higher the TSI value, the less stable the emulsion is.

\subsection{Analysis of in vitro lipid digestion}

This study used a modified form of a previously-described in vitro digestion model ${ }^{42)}$. The samples, containing $175 \mathrm{mg}$ MCT, were placed in a glass beaker containing $30 \mathrm{~mL}$ buffer solution (10 mmol/L Tris-HCl, $\mathrm{pH}$ 7.0) and incubated at $37^{\circ} \mathrm{C}$ for $15 \mathrm{~min}$. Then, $2.5 \mathrm{~mL}$ of NaCh, $1.5 \mathrm{~mL}$ of $\mathrm{CaCl}_{2}$ solution and $1 \mathrm{~mL}$ of $\mathrm{NaCl}$ solution were added to all the samples (except for control group to which $\mathrm{NaCh}$ was not added) with continuous stirring and the $\mathrm{pH}$ was adjusted to 7 . Then, $1.5 \mathrm{~mL}$ of freshly prepared pancreatic lipase suspension was added to the mixture. The final digestion medium contained $175 \mathrm{mg}$ lipid, $150 \mathrm{mmol} / \mathrm{L} \mathrm{NaCl}$, $20 \mathrm{mmol} / \mathrm{L} \mathrm{CaCl}_{2}, 5 \mathrm{mg} / \mathrm{mL} \mathrm{NaCh}$, and $1.6 \mathrm{mg} / \mathrm{mL}$ pancreatic lipase. A pH-stat automatic titration unit (916 Ti-Touch Metrohm, Switzerland)was used to automatically monitor the $\mathrm{pH}$ and maintain it at 7.0 by titrating appropriate amount of $\mathrm{NaOH}$ solution to neutralize the release of any free fatty acids (FFAs). The volume of $\mathrm{NaOH}$ added to the samples was recorded. The percentage of FFAs released was calculated using Eq. (4), as following:

$$
\operatorname{FFA}(\%)=100 \times \frac{V_{\mathrm{NaOH}} \times m_{\mathrm{NaOH}} \times M_{\text {lipid }}}{W_{\text {lipid }} \times 2}
$$

where, $V_{\mathrm{NaOH}}$ is the volume of $\mathrm{NaOH}$ required for neutraliz- 
ing the FFA released $(\mathrm{mL}) ; m_{\mathrm{NaOH}}$ is the molarity of $\mathrm{NaOH}$ solution used $(0.1 \mathrm{~mol} / \mathrm{L}) ; W_{\text {lipid }}$ is the total weight of oil present initially in the reaction vessel; and $M_{\text {lipid }}$ is the average molecular weight of MCT $(509.44 \mathrm{~g} / \mathrm{mol})$.

\subsection{Statistical analysis}

All the experiments were performed in duplicates, with at least three measurements being made per sample.

\section{Results and Discussion}

\subsection{Interfacial dilational rheology}

3.1.1 Dynamic interfacial tension and dilational modulus

The dynamic IFTs and dilational moduli for the interfac-
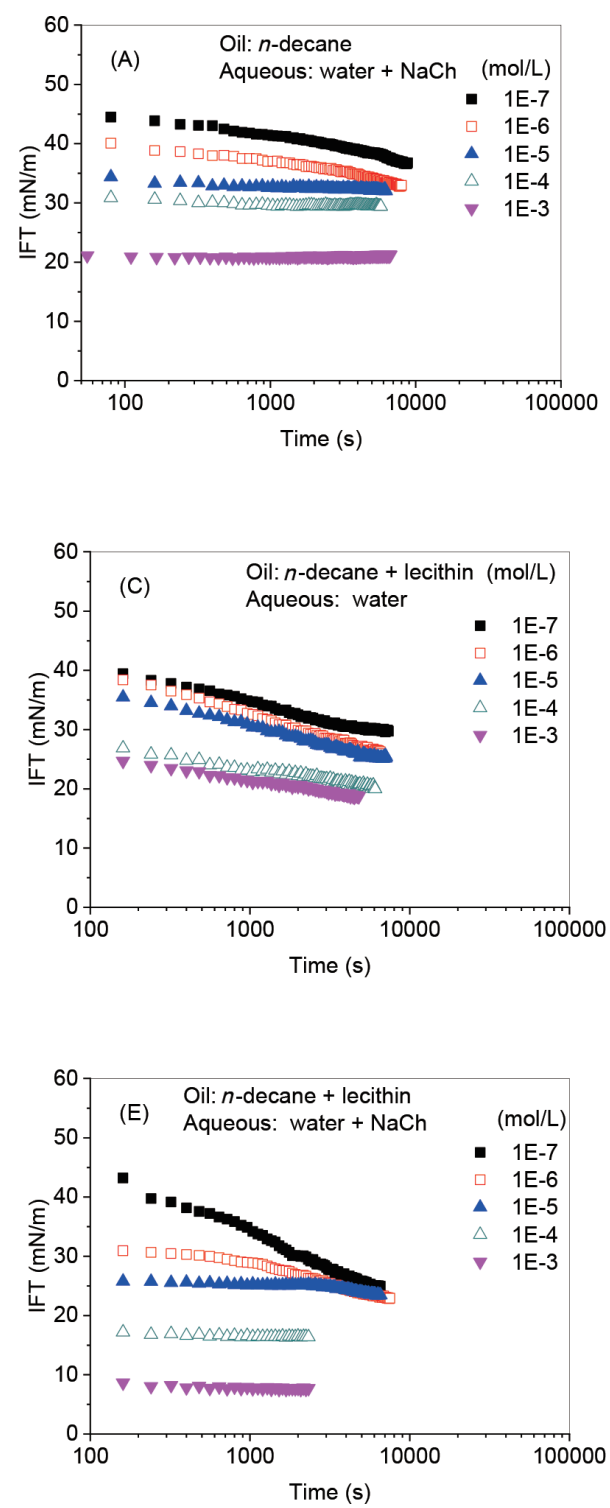

es of pure oil-NaCh aqueous solution, lecithin oil solutionpure water, and lecithin oil solution-NaCh aqueous solution are presented in Fig. 1. Generally, when a new interface is created, the amphiphiles in the bulk phase start to adsorb at the interface, leading to the decrease in IFT. As the interfacial concentration of amphiphiles increases, the deformation in interface causes higher IFT gradient, leading to the increase in interfacial dilational modulus. When the amphiphiles reach an equilibrium adsorption, both the IFT and dilational modulus become constants ${ }^{43)}$. The dynamic IFTs and interfacial dilational moduli for the three systems in this study followed similar trends as that of general amphiphiles. At low concentrations, the diffusion rate of amphiphiles from the bulk to interface was low, so the IFT decreased slowly. With the increase in the concentration of
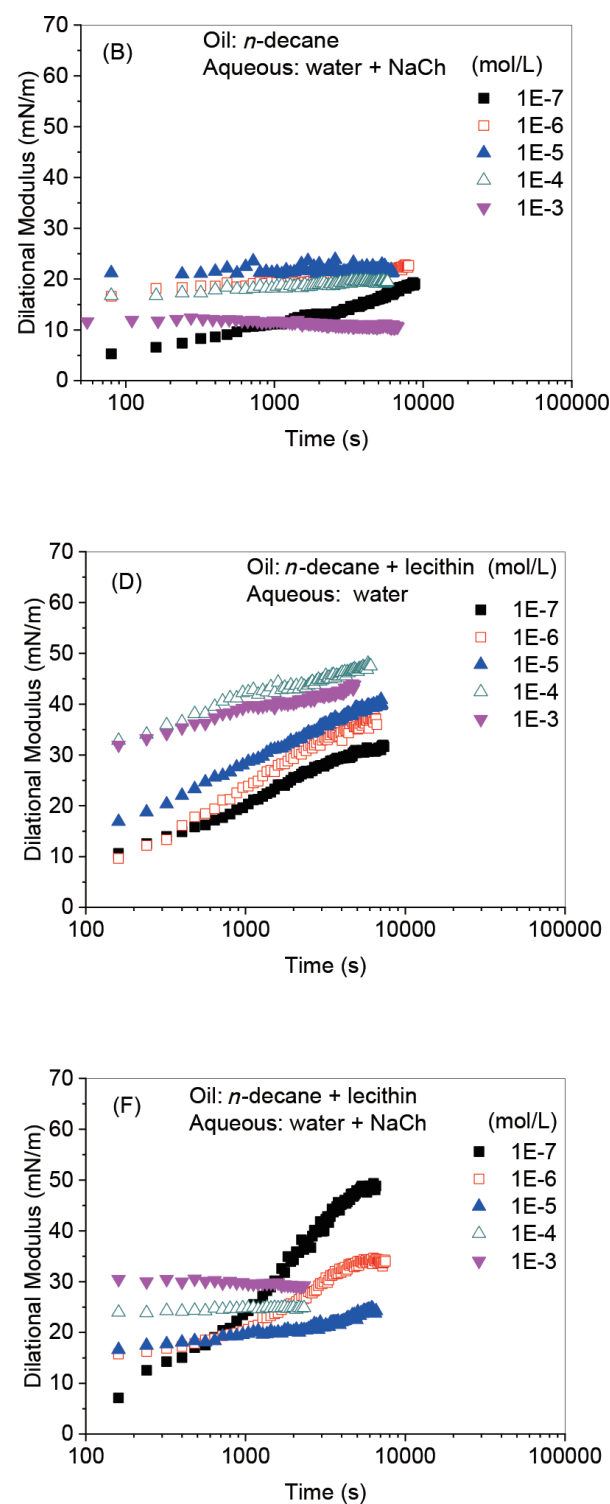

Fig. 1 Dynamic IFTs and dilational moduli for the interfaces of pure oil-NaCh aqueous solution (A, B), lecithin oil solutionpure water (C, D), and lecithin oil solution-NaCh aqueous solution (E, F). 


\section{Bile Salts Accelerate the Diffusion Exchange of Materials between the Bulk and Interface}

amphiphiles, their diffusion also accelerated. For NaCh at higher concentrations $\left(\geq 1 \times 10^{-5} \mathrm{~mol} / \mathrm{L}\right)$, IFTs and dilational moduli could quickly reach equilibrium values. But for lecithin at high concentration $\left(1 \times 10^{-5} \mathrm{~mol} / \mathrm{L} \sim 1 \times 10^{-3}\right.$ $\mathrm{mol} / \mathrm{L}$ ), the IFTs still decreased slowly during the interfacial adsorption, indicating slower diffusion rate than that of $\mathrm{NaCh}$. But lecithin could reduce the final IFT to a lower level than that of $\mathrm{NaCh}$ at the same concentrations. For the interface between the lecithin oil solution and the $\mathrm{NaCh}$ aqueous solution, the IFT could be further decreased while still maintaining a rapid adsorption rate.

3.1.2 Concentration dependence of interfacial tension and dilational modulus

The equilibrium IFTs and interfacial dilational moduli for the three systems at different concentrations are presented in Fig. 2. As shown in Fig. 2(A), the IFTs between lecithin oil solutions and pure water were lower than those of between pure oil and $\mathrm{NaCh}$ aqueous solutions at the same concentrations. This indicated that lecithin had higher interfacial activity. Moreover, Fig. 2(B) also shows that the dilational moduli for lecithin were also dramatically higher than those of $\mathrm{NaCh}$.

As is known to all, NaCh exhibits planar polarity. Given this special structure, NaCh molecules tend to lie on the interface with the concave side faces the aqueous phase while the convex side faces the oil phase. Therefore, it is unlikely for the NaCh molecules to be closely packed and thereby exhibit weak intermolecular interactions. As to lecithin, the major component, phosphatidylcholine, is composed of a polar head-group containing phosphocholine and glycerol residue ${ }^{44)}$. Its polar group bears both positive and negative charges, which may generate strong electrostatic attraction. Moreover, the long hydrocarbon chains of phospholipids enhance van der Waals forces between hydrophobic groups. The high dilational modulus and low IFT indicated the formation of a compact arrangement of adsorption film under strong intermolecular interactions.

At the interface between lecithin oil solution and $\mathrm{NaCh}$ aqueous solution, both the lecithin and $\mathrm{NaCh}$ tended to

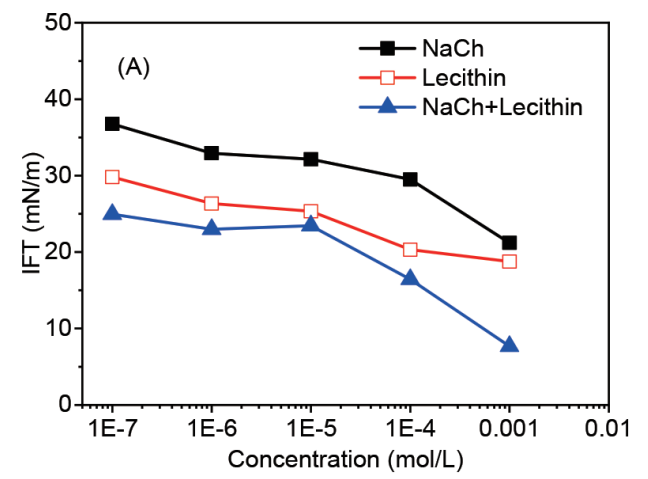

adsorb to the interface. As shown in Fig. 2(A), the IFT dropped to lower values than individual lecithin and $\mathrm{NaCh}$. For example, at the concentration of $1 \times 10^{-3} \mathrm{~mol} / \mathrm{L}$, the individual $\mathrm{NaCh}$ and lecithin decreased IFTs to $21.2 \mathrm{mN} / \mathrm{m}$ and $18.8 \mathrm{mN} / \mathrm{m}$, respectively, while that of lecithin oil solution and $\mathrm{NaCh}$ aqueous solution decreased to $7.7 \mathrm{mN} / \mathrm{m}$. This signified that NaCh was mixed adsorbed with lecithin at interface and they showed a good synergistic effect in decreasing IFT. Tung et al. used infrared spectroscopy for studying the interactions between bile salts and lecithin in oil phase. They found hydrogen bonding interactions between phosphate groups of lecithin and hydroxyl groups of $\mathrm{NaCh}$ played a significant role in the formation of reverse wormlike micelles ${ }^{45)}$. The hydrogen bonding interactions might also be a driving force for their assembly at the interface. Njauw et al. reported that the bile salts could be adsorbed onto phospholipid monolayers and decrease the IFT to lower level than that determined by the bile salts alone. This suggested a certain synergistic interaction of bile salts with the adsorbed-lipid layer ${ }^{46)}$.

The amount of amphiphiles adsorbed at interface increases with the increase in bulk concentration until it reaches critical micelle concentrations (CMC). Therefore, it enhances intermolecular interactions at the interface and causes higher IFT gradients during the interfacial compression and expansion. On the other hand, increase in the bulk concentration of amphiphiles also increases the molecular diffusion exchange between the bulk and interface, which can quickly reduce the IFT gradient and lead to a decrease in the dilational modulus ${ }^{47)}$. Generally, in the low concentration range, the increase in the amount of amphiphiles adsorbed at the interface is the decisive factor that determines the interfacial dilational modulus. In the high concentration range, the diffusion exchange of amphiphile molecules between the bulk and interface is enhanced and becomes dominant in determining the dilational modulus. Under these two opposite effects, the dilational modulus increases in the range of low concentration and decreases in the range of high concentration, and a maximal dilational

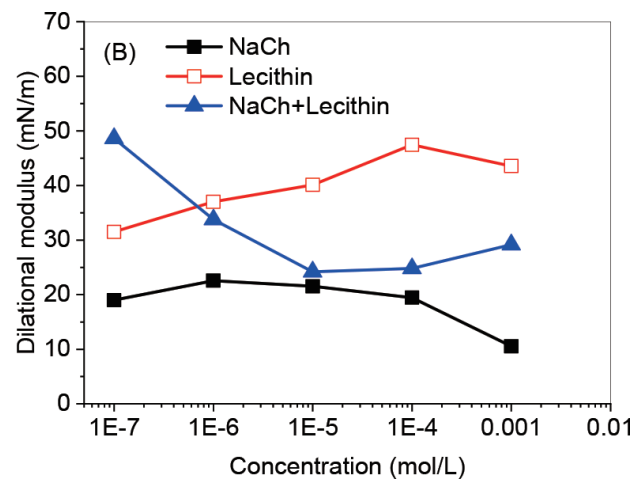

Fig. 2 Equilibrium IFTs (A) and interfacial dilational moduli(B) at the $n$-decane-aqueous interfaces as a function of concentration for $\mathrm{NaCh}$, lecithin, and $\mathrm{NaCh}-\mathrm{lecithin}$ mixed adsorption layers. 
modulus appears with the increase in the amphiphile concentration $^{48}$. For systems with negligible molecular diffusion exchange, even when the concentration reaches CMC, the interface dilational modulus will not decrease significantly, while, for the systems where diffusion exchange is very significant, the maximal dilational modulus even appears at a much lower concentration. Interestingly, for the individual $\mathrm{NaCh}$ and lecithin, and their combined adsorption layer, the interfacial dilational moduli as a function of concentration exhibited different trends. The curve of interfacial dilational modulus vs. concentration for $\mathrm{NaCh}$ was consistent with that of the general surfactants and reached the maximum $(22.6 \mathrm{mN} / \mathrm{m})$ at the concentration of $1 \times 10^{-6} \mathrm{~mol} / \mathrm{L}$. At higher concentrations, the diffusion exchange became significant, and thus the dilational modulus started to decrease. For individual lecithin, the dilational modulus kept increasing with the increase in concentration and decreased slightly after reaching maximum $(47.5 \mathrm{mN} /$ $\mathrm{m})$ at $1 \times 10^{-4} \mathrm{~mol} / \mathrm{L}$, which suggested that the diffusion exchange of lecithin was insignificant. Apart from that, because of the strong interactions between lecithin molecules, the dilational moduli were dramatically higher than those of NaCh. Particularly, at high concentrations, the lecithin molecules were closely arranged at the interface under strong intermolecular interactions, which further inhibited the diffusion exchange. As to NaCh-lecithin mixed interface, the dilational modulus was as high as $48.6 \mathrm{mN} / \mathrm{m}$ at the concentration of $1 \times 10^{-7} \mathrm{~mol} / \mathrm{L}$, suggesting a strong synergy between these two amphiphiles. However, with the increase of concentration, the dilational modulus started decreasing. This unique variation indicated that, even the amphiphiles had generated an adsorption film with high dilational modulus, the molecules at interface were not closely packed. As a result, the diffusion exchange easily occurred and became the dominant factor at such low concentrations. Moreover, lecithin and $\mathrm{NaCh}$ diffused to the interface from oil phase and aqueous phase, respectively, which made the diffusion exchange effect more significant. 3.1.3 Frequency dependence of the interfacial dilational rheological properties

The viscoelasticity of interfacial films can be described from the dependence of dilational rheological parameters on oscillating frequency. Herein, the effect of oscillating frequency on the dilational elasticity and viscosity for the individual $\mathrm{NaCh}$ and lecithin, and their interfacial mixtures were measured and the results are shown in Fig. 3.

As shown in Fig. 3, the dilational elasticity for the three systems increased with the increase in oscillation frequency. At low frequencies, the amphiphiles have enough time to restore the changes in IFT, caused by the interfacial deformation, through relaxation processes, such as the diffusion exchange of amphiphile molecules between the bulk and interface and their reorientation and rearrangement. As a result, the dilational elasticity is lower at low oscilla- tion frequency. On the other hand, the rapid deformation rate of interface at high frequencies leaves insufficient time for the restoration of interfacial film after deformation, leading to a higher dilational elasticity at a high oscillation frequency. In the moderate oscillation frequency range, the dilational elasticity increases with the increase in the oscillation frequency.

The dilational viscosity is believed to be related to various relaxation processes, occurring at or near the interface. When the interface deforms at low frequencies, the IFT gradient caused by interface deformation can be completely eliminated within the experimental time, and the dilational viscosity is close to 0 . At extremely higher frequencies, the interface deformation rate exceeds the characteristic frequencies of relaxation processes. Therefore, no relaxation processes occur and the dilational viscosity is also close to 0 . In the moderate frequency range, the viscosity spectrum will exhibit maxima at the characteristic frequencies of the relaxation processes ${ }^{49)}$. As shown in Fig. 3(B), the dilational viscosity of individual $\mathrm{NaCh}$ increased monotonically with the increase in frequency, meaning that the maximum dilational viscosity would appear at a frequency higher than the highest oscillation frequency $(0.1$ $\mathrm{Hz}$ ) used in this study. This indicated that there was a rapid relaxation process at or near the NaCh-adsorbed interfacial film, such as the diffusion exchange of amphiphilic molecules. For individual lecithin, as shown in Fig. 3(D), the dilational viscosity decreased gradually with the increase in oscillation frequency, meaning that the maximum dilational viscosity would appear at a frequency lower than the lowest oscillation frequency used in this $\operatorname{study}(0.005 \mathrm{~Hz})$. This suggested that the slow relaxation processes, such as re-orientation or rearrangement, played a decisive role in determining the interfacial dilational properties. However, the mixed absorption layer of lecithin with $\mathrm{NaCh}$ showed an increasing trend for dilational viscosity with the increase in oscillation frequency within the tested frequency range. This means that, like single NaCh adsorption layer, the characteristic frequency of dominate relaxation process at the mixed adsorption layer was higher than the highest oscillating frequency $(0.1 \mathrm{~Hz})$ used in the present work. As compared to the individual lecithin layer, the relaxation processes of the lecithin-NaCh composite interface have been accelerated significantly, which enhanced the interfacial viscoelasticity.

3.1.4 Interfacial dilational modulus as a function of interfacial pressure

As reported, for irreversibly adsorbed interfacial films, such as proteins and amphiphiles with strong intermolecular interactions, their interfacial properties are dominated by the amount and intermolecular interactions of the amphiphile molecules at the interface, which can be quantitatively expressed as their interfacial pressure. Therefore, the dilational modulus at various adsorption time and bulk 

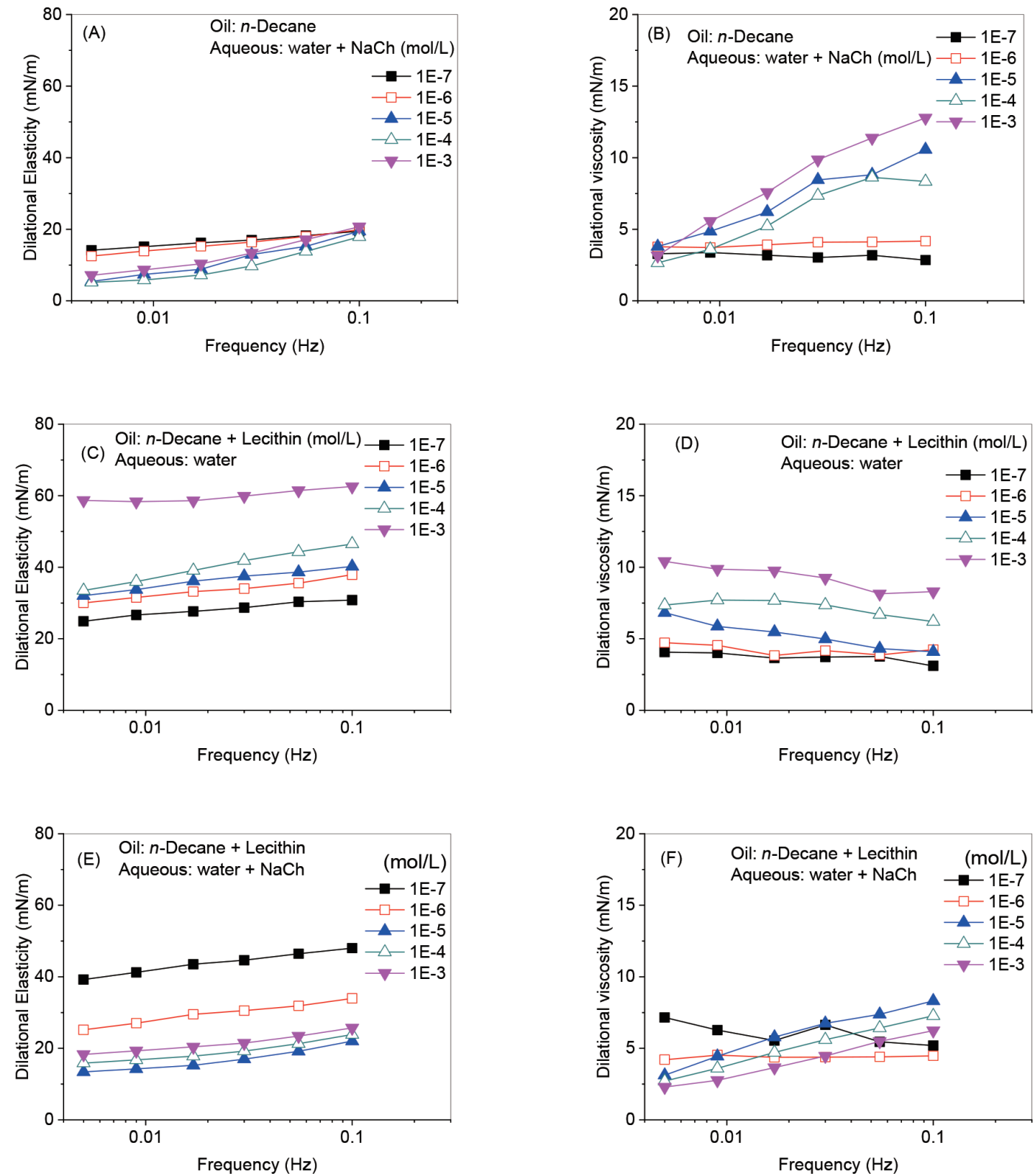

Fig. 3 Interfacial dilational elasticity and viscosity for $\mathrm{NaCh}(\mathrm{A}, \mathrm{B})$, lecithin (C, D), and NaCh-lecithin mixed film (E, F) as a function of frequency.

concentrations coincides on a single dilational modulus-interfacial pressure curve ${ }^{47,50-53)}$. On the contrary, the interfacial properties of conventional surfactants are mainly dominated by the molecular diffusion exchange between the bulk and interface at high concentrations. Therefore, at a fixed interfacial pressure, higher the concentration of surfactant in bulk phase, faster will be the diffusion exchange of surfactant molecules between the bulk and interface. As a result, the IFT gradient caused by oscillation can be quickly reduced, leading to lower interfacial dilational modulus. That is, the dilational modulus-interfacial pressure curves of different concentrations are scattered instead of overlapping ${ }^{54,55)}$.
The dilational modulus vs interfacial pressure curves for individual $\mathrm{NaCh}$ and lecithin, and their interfacial mixture at various concentrations are presented in Fig. 4. As shown in Fig. 4(A), the curves of $\mathrm{NaCh}$ at different concentrations could be divided into two groups. At low NaCh concentrations $\left(\leq 1 \times 10^{-5} \mathrm{~mol} / \mathrm{L}\right)$, these curves coincided with each other, indicating that the diffusion exchange of $\mathrm{NaCh}$ molecules between bulk and interface was inappreciable. However, with the increase of amphiphile concentration, the diffusion exchange became increasingly significant. When the NaCh concentration was higher than $1 \times 10^{-5}$ $\mathrm{mol} / \mathrm{L}$, the curves started to separate from being overlapped, suggesting that the diffusion exchange of amphi- 

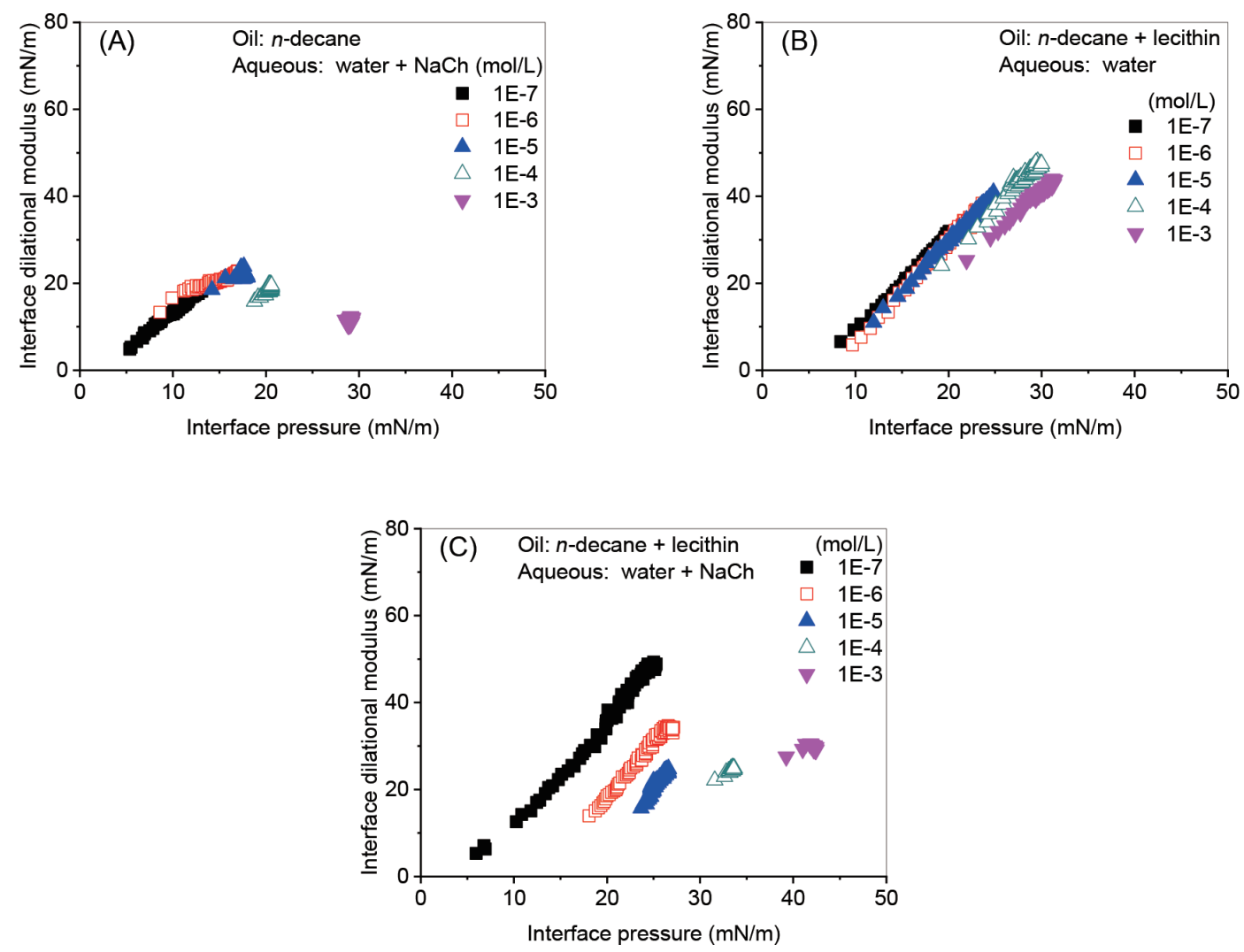

Fig. 4 Interfacial dilational modulus for individual $\mathrm{NaCh}(\mathrm{A})$ and lecithin (B) and NaCh-lecithin mixed layer (C) as a function of interfacial pressure.

philes between the interface and bulk solution began to dominate the nature of the adsorption layer. However, for the individual lecithin, as shown in Fig. 4(B), the dilational modulus vs interfacial curves were well-overlapped, indicating that the interfacial properties were dominated by the in-interface processes, while the diffusion exchange was negligible. The main relaxation processes of the lecithin interfacial films might only be the slow processes within the interface, such as the reorientation and rearrangement of amphiphile molecules, which are consistent with the low characterization frequencies as inferred from Fig. 3(D). As to the NaCh-lecithin mixed layers, the curves of dilational modulus vs. interfacial pressure at different concentrations were separated from each other, implying the fast relaxation process, diffusion exchange, has become the dominant factor, which further confirmed the speculation in Section 3.1.3.

\subsection{Emulsion properties}

In order to evaluate the emulsion properties, $\mathrm{NaCh}$ and lecithin were dissolved in water and MCT, respectively, and the aqueous and oil phases were mixed to prepare the $\mathrm{NaCh}$-lecithin emulsions. The concentration of $\mathrm{NaCh}$ ranged from 0 to $1.5 \mathrm{wt} \%$, while that of lecithin was fixed at $0.5 \mathrm{wt} \%$. The emulsion stabilized only by $\mathrm{NaCh}$ was also prepared and used as a control. Figure $5(\mathrm{~A})$ presents the effects of $\mathrm{NaCh}$ on the droplet size of the lecithin-stabilized emulsions. The size of emulsion droplets stabilized only with $0.5 \mathrm{wt} \%$ lecithin was around $600 \mathrm{~nm}$. NaCh-stabilized emulsions had smaller droplets with the average size of around $160 \mathrm{~nm}$ at the same concentration. When $\mathrm{NaCh}$ and lecithin were introduced into water and oil phases separately to prepare emulsions, the droplets size was comparable to that of $\mathrm{NaCh}$-stabilized emulsions. With the increase of NaCh concentration from 0.1 wt\% to $1.5 \mathrm{wt} \%$, a moderate decrease in the mean droplet size was observed. As discussed before, $\mathrm{NaCh}$ exhibited faster diffusion behavior from the bulk to interface. As a result, an adsorption layer was quickly formed on the interface during emulsification, thereby contributing to the better emulsifying ability of $\mathrm{NaCh}$. Even in the presence of lecithin, $\mathrm{NaCh}$ played a leading role in the process of emulsification.

Figure 5(B) shows the destabilization kinetics of emulsions stabilized with the individual $\mathrm{NaCh}$ and lecithin, and their interfacial mixtures. Figure 6 shows the backscattering light intensity $(\mathrm{BS})$ of lecithin and $\mathrm{NaCh}$ emulsion as a function of time and sample height. It can be seen from Fig. 6(A) that the BS curves kept almost unchanged with either the time or the sample height for the emulsion stabilized by lecithin. As discussed in 3.1, lecithin formed a 

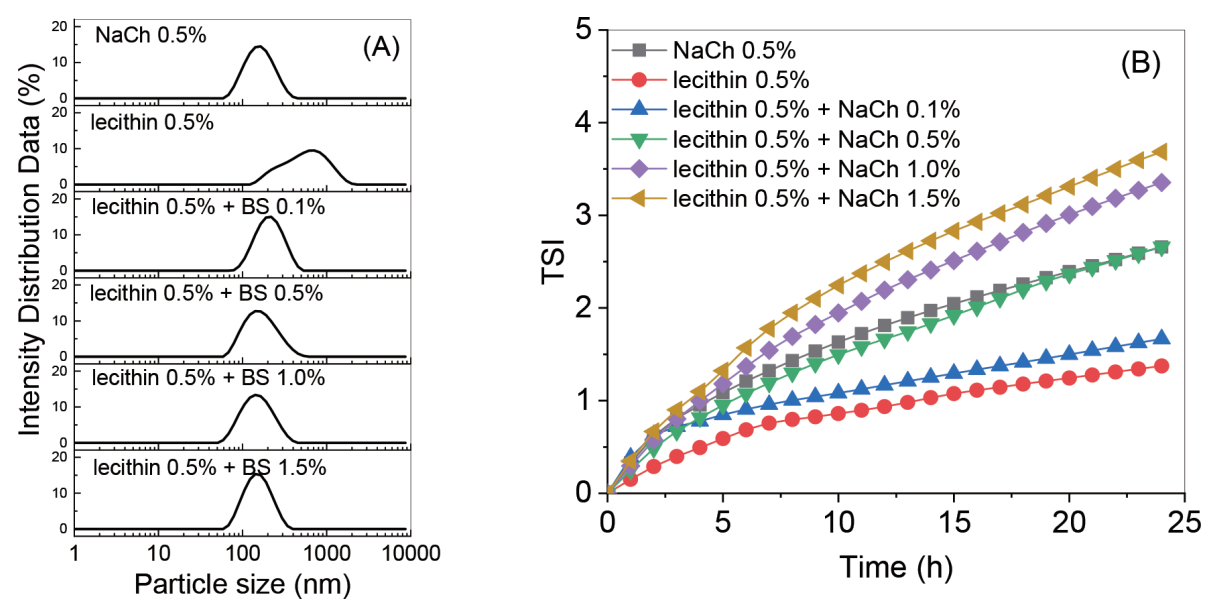

Fig. 5 Oil droplets size(A) and stability of emulsion(B) stabilized with NaCh and/or lecithin.
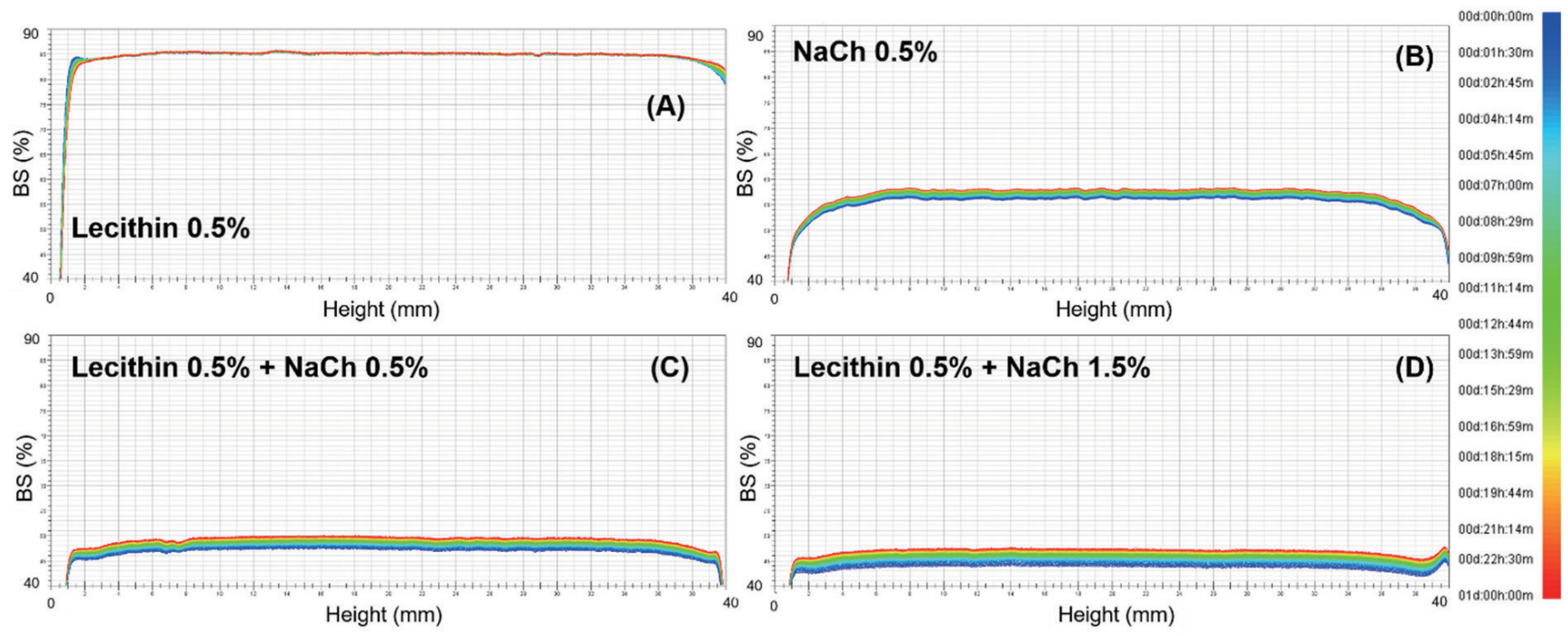

Fig. 6 Variation of BS of O/W emulsions stabilized by (A) lecithin, (B) NaCh, and (C, D) NaCh-lecithin mixed layer.

tightly arranged film on the interface, thereby exhibiting better emulsion stability. On the contrary, due to the loose arrangement of $\mathrm{NaCh}$ molecules at the interface, the emulsion stabilized by $\mathrm{NaCh}$ was less stable as shown in Fig. 5 (B). Figure 6 (B) shows that the BS of NaCh emulsion decreased uniformly at different sample heights, indicating the increase of average droplet size due to the flocculation and coalescence of emulsion droplets. As to the emulsions stabilized by lecithin-NaCh mixtures shown in Figs. 6C and $6 \mathrm{D}$, the BS still decreased uniformly for the entire sample height range, meaning that destabilization of emulsion was mainly due to droplet flocculation and coalescence. For the lecithin-NaCh mixed adsorbed layers, higher the concentration of $\mathrm{NaCh}$ in bulk, greater would be the influence of bile salts on the interface films and worse would be the stability of emulsions.

\subsection{In vitro digestion fate of emulsions}

The in vitro digestion fate of emulsions stabilized by lecithin was measured and the digestion kinetics is shown in Fig. 7. During the in vitro digestion in the absence of $\mathrm{NaCh}$, the lecithin molecules covered the oil-aqueous interface and formed a compact adsorption film, which greatly hindered the molecular diffusion exchange between the bulk and the interface. Therefore, it blocked the contact of lipase with triacylglycerols. As a result, the digestion rate was dramatically slow and digestion extend was as low as $40 \%$. However, when NaCh was added during the digestion, due to the fast diffusion exchange of NaCh between bulk and interface, it quickly entered the interface and formed a mixed adsorption layer with lecithin. As shown in the results of interface dilatational rheology in Section 3.1, the viscoelasticity of interface increased, making the material exchange between the bulk and interface easier to take places. This created suitable conditions for the binding of lipases to lipid droplets. Therefore, the lipid digestion became faster and the digestion extend was significantly promoted. 


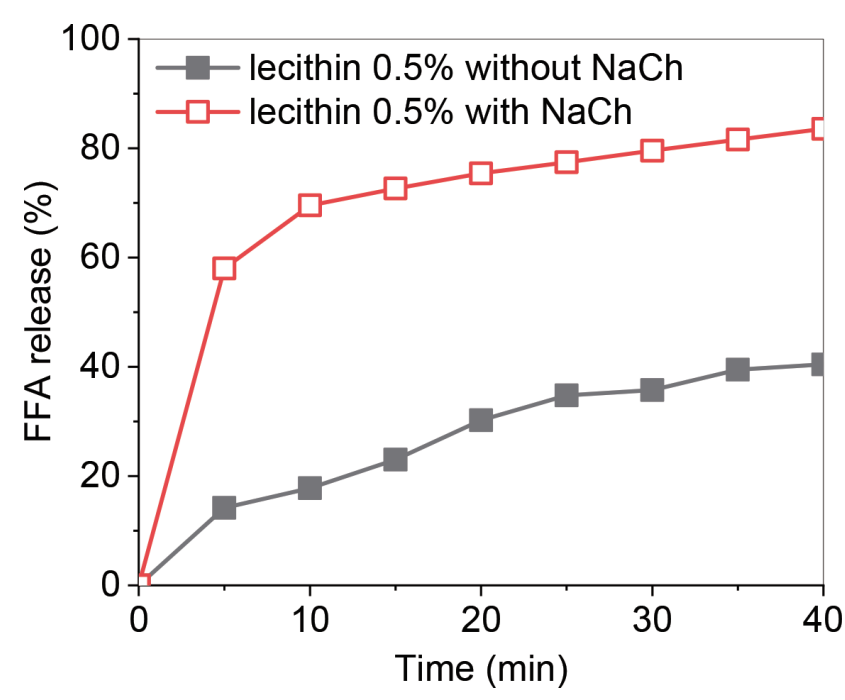

Fig. 7 Digestion kinetics of lipid emulsion stabilized by lecithin in the absence and presence of $\mathrm{NaCh}$.

\section{Conclusions}

The current study focused on the role of bile salts in lipid digestion from the perspective of interfacial viscoelasticity and relaxation processes. $\mathrm{NaCh}$ and lecithin were selected as model compounds for bile salts and food emulsifiers, respectively. The interfacial dilational rheological behaviors, emulsion properties, and in vitro digestion fate of lipids were characterized. The experimental results showed that the lecithin alone formed a tightly-arranged elastic layer at the interface, thereby reducing the diffusion exchange of molecules between the bulk and interface. This interfacial feature helped to form a stable interfacial film, which enhanced the stability of emulsion and hindered the accessibility of lipases to lipids. Due to the special polar surface structure of NaCh, they could not form tightly-arranged layers at the interface. However, the diffusion exchange of NaCh molecules between bulk and interface was significant, suggesting that they could quickly enter the interface and replace the lecithin molecules. As a result, the lecithin interfacial adsorption layer, which was originally dominated by the slow relaxation processes within the interface, was transformed into one controlled by molecular diffusion exchange process. This transition accelerated the material exchange between the bulk and interface, thus creating suitable conditions for the interfacial adsorption of lipases, and promoting the digestion process. These results provided a deeper understanding of the interfacial behavior of bile salts with emulsifiers, which would provide a basis for the rational design of interfacial layer to modulate lipid digestion.

\section{Acknowledgements}

This work was financially supported by the National
Natural Science Foundation of China(22008002), Beijing Municipal Education Commission(KM202110011001), and National Key R\&D Program of China(2017YFB0308700).

\section{References}

1) Reis, P.; Holmberg, K.; Debeche, T.; Folmer, B.; Fauconnot, L.; Watzke, H. Lipase-catalyzed reactions at different surfaces. Langmuir 22, 8169-8177 (2006).

2) Guo, Q.; Ye, A.; Bellissimo, N.; Singh, H.; Rousseau, D. Modulating fat digestion through food structure design. Prog. Lipid Res. 68, 109-118(2017).

3) Maljaars, P.; Symersky, T.; Kee, B.; Haddeman, E.; Peters, H.; Masclee, A. Effect of ileal fat perfusion on satiety and hormone release in healthy volunteers. Int. $J$. Obes. 32, 1633-1639 (2008).

4) Maljaars, P.; Peters, H.; Mela, D.; Masclee, A. Ileal brake: A sensible food target for appetite control. A review. Physiol. Behav. 95, 271-281 (2008).

5) Maldonado-Valderrama, J. Probing in vitro digestion at oil-water interfaces. Curr. Opin. Colloid Interface Sci. 39, 51-60 (2019).

6) Macierzanka, A.; Torcello-Gomez, A.; Jungnickel, C.; Maldonado-Valderrama, J. Bile salts in digestion and transport of lipids. Adv. Colloid Interface Sci. 274, 17 (2019).

7) Maldonado-Valderrama, J.; Wilde, P.; Macierzanka, A.; Mackie, A. The role of bile salts in digestion. Adv. Colloid Interface Sci. 165, 36-46 (2011).

8) Golding, M.; Wooster, T.J. The influence of emulsion structure and stability on lipid digestion. Curr. Opin. Colloid Interface Sci. 15, 90-101 (2010).

9) Mukherjee, M. Human digestive and metabolic lipases-a brief review. J. Mol. Catal. B: Enzym. 22, 369376 (2003).

10) Armand, M. Lipases and lipolysis in the human digestive tract: where do we stand? Curr. Opin. Clin. Nutr. Metab. Care 10, 156-164(2007).

11) Gu, Y.S.; Decker, E.A.; McClements, D.J. Influence of $\mathrm{pH}$ and carrageenan type on properties of $\beta$-lactoglobulin stabilized oil-in-water emulsions. Food Hydrocoll. 19, 83-91 (2005).

12) Wilde, P.; Chu, B. Interfacial \& colloidal aspects of lipid digestion. Adv. Colloid Interface Sci. 165, 14-22 (2011).

13) Mun, S.; Decker, E.A.; McClements, D.J. Influence of emulsifier type on in vitro digestibility of lipid droplets by pancreatic lipase. Food Res. Int. 40, 770-781 (2007).

14) Torcello-Gómez, A.; Jódar-Reyes, A.; MaldonadoValderrama, J.; Martín-Rodríguez, A. Effect of emulsifier type against the action of bile salts at oil-water interfaces. Food Res. Int. 48, 140-147(2012). 


\section{Bile Salts Accelerate the Diffusion Exchange of Materials between the Bulk and Interface}

15) Jódar-Reyes, A.; Torcello-Gómez, A.; Wulff-Pérez, M.; Gálvez-Ruiz, M.; Martín-Rodríguez, A. Different stability regimes of oil-in-water emulsions in the presence of bile salts. Food Res. Int. 43, 1634-1641 (2010).

16) Bellesi, F.A.; Ruiz-Henestrosa, V.M.P.; Pilosof, A.M. Behavior of protein interfacial films upon bile salts addition. Food Hydrocoll. 36, 115-122 (2014).

17) Torcello-Gomez, A.; Foster, T.J. Influence of interfacial and bulk properties of cellulose ethers on lipolysis of oil-in-water emulsions. Carbohydr. Polym. 144, 495503 (2016).

18) Ruiz-Henestrosa, V.M.P.; Bellesi, F.A.; Camino, N.A.; Pilosof, A.M. The impact of HPMC structure in the modulation of in vitro lipolysis: The role of bile salts. Food Hydrocoll. 62, 251-261 (2017).

19) Espinal-Ruiz, M.; Parada-Alfonso, F.N.; RestrepoSánchez, L.P.; Narváez-Cuenca, C.E.; McClements, D.J. Interaction of a dietary fiber (pectin) with gastrointestinal components (bile salts, calcium, and lipase): A calorimetry, electrophoresis, and turbidity study. $J$. Agric. Food Chem. 62, 12620-12630 (2014).

20) Torcello-Gómez, A.; Fraguas, C.F.; Ridout, M.J.; Woodward, N.C.; Wilde, P.J.; Foster, T.J. Effect of substituent pattern and molecular weight of cellulose ethers on interactions with different bile salts. Food Funct. 6, 730-739 (2015).

21) Wei, Y.; Tong, Z.; Dai, L.; Ma, P.; Zhang, M.; Liu, J.; Mao, L.; Yuan, F.; Gao, Y. Novel colloidal particles and natural small molecular surfactants co-stabilized Pickering emulsions with hierarchical interfacial structure: Enhanced stability and controllable lipolysis. J. Colloid Interface Sci. 563, 291-307 (2020).

22) Rodrigues Costa, A.L.; Gomes, A.; Furtado, G.d.F.; Tibolla, H.; Menegalli, F.C.; Cunha, R.L. Modulating in vitro digestibility of Pickering emulsions stabilized by food-grade polysaccharides particles. Carbohydr. Polym. 227, 115344(2020).

23) Jodar-Reyes, A.B.; Torcello-Gomez, A.; Wulff-Perez, M.; Galvez-Ruiz, M.J.; Martin-Rodriguez, A. Different stability regimes of oil-in-water emulsions in the presence of bile salts. Food Res. Int. 43, 1634-1641 (2010).

24) Torcello-Gomez, A.; Jodar-Reyes, A.B.; MaldonadoValderrama, J.; Martin-Rodriguez, A. Effect of emulsifier type against the action of bile salts at oil-water interfaces. Food Res. Int. 48, 140-147(2012).

25) Koelsch, P.; Motschmann, H. Relating foam lamella stability and surface dilational rheology. Langmuir 21, 6265-6269 (2005).

26) Lexis, M.; Willenbacher, N. Relating foam and interfacial rheological properties of $\beta$-lactoglobulin solutions. Soft Matter 10, 9626-9636(2014).

27) Fruhner, H.; Wantke, K.-D.; Lunkenheimer, K. Relationship between surface dilational properties and foam stability. Colloids Surf. A 162, 193-202(2000).
28) Acharya, D.P.; Gutiérrez, J.M.; Aramaki, K.; Aratani, K.-i.; Kunieda, H. Interfacial properties and foam stability effect of novel gemini-type surfactants in aqueous solutions. J. Colloid Interface Sci. 291, 236-243 (2005).

29) Stubenrauch, C.; Fainerman, V.; Aksenenko, E.; Miller, R. Adsorption behavior and dilational rheology of the cationic alkyl trimethylammonium bromides at the water/air interface. J. Phys. Chem. B 109, 1505-1509 (2005).

30) Wantke, K.-D.; Örtegren, J.; Fruhner, H.; Andersen, A.; Motschmann, H. The influence of the sublayer on the surface dilatational modulus. Colloids Surf. A 261, 75-83 (2005).

31) Zamora, J.M.; Marquez, R.; Forgiarini, A.M.; Langevin, D.; Salager, J.-L. Interfacial rheology of low interfacial tension systems using a new oscillating spinning drop method. J. Colloid Interface Sci. 519, 27-37 (2018).

32) Torcello-Gomez, A.; Maldonado-Valderrama, J.; de Vicente, J.; Cabrerizo-Vilchez, M.A.; Galvez-Ruiz, M.J.; Martin-Rodriguez, A. Investigating the effect of surfactants on lipase interfacial behaviour in the presence of bile salts. Food Hydrocoll. 25, 809-816 (2011).

33) Cui, L.; Decker, E.A. Phospholipids in foods: Prooxidants or antioxidants? J. Sci. Food Agric. 96, 18-31 (2016).

34) Küllenberg, D.; Taylor, L.A.; Schneider, M.; Massing, U. Health effects of dietary phospholipids. Lipids Health Dis. 11, 3(2012).

35) Pichot, R.; Watson, R.L.; Norton, I.T. Phospholipids at the interface: Current trends and challenges. Int. J. Mol. Sci. 14, 11767-11794(2013).

36) Mottola, M.; Vico, R.V.; Villanueva, M.E.; Fanani, M.L. Alkyl esters of l-ascorbic acid: Stability, surface behaviour and interaction with phospholipid monolayers. $J$. Colloid Interface Sci. 457, 232-242(2015).

37) Evans, D.F.; Wennerström, H. The colloidal domain: Where physics, chemistry, biology, and technology meet. 1999.

38) Hofmann, A.; Small, D. Detergent properties of bile salts: Correlation with physiological function. Annu. Rev. Med. 18, 333-376 (1967).

39) Carey, M.C.; Small, D.M. The characteristics of mixed micellar solutions with particular reference to bile. Am. J. Med. 49, 590-608(1970).

40) Krägel, J.; Li, J.; Miller, R.; Bree, M.; Kretzschmar, G.; Möhwald, H. Surface viscoelasticity of phospholipid monolayers at the air/water interface. Colloid Polym. Sci. 274, 1183-1187(1996).

41) Trujillo-Cayado, L.A.; Alfaro, M.C.; Muñoz, J.; Raymundo, A.; Sousa, I. Development and rheological properties of ecological emulsions formulated with a biosolvent and two microbial polysaccharides. Colloids Surf. B 141, 53-58(2016). 
42) Mun, S.; Decker, E.A.; Park, Y.; Weiss, J.; McClements, D.J. Influence of interfacial composition on in vitro digestibility of emulsified lipids: Potential mechanism for chitosan's ability to inhibit fat digestion. Food Biophys. 1, 21-29 (2006).

43) Cao, X.-L.; Feng, J.; Guo, L.-L.; Zhu, Y.-w.; Zhang, L.; Zhang, L.; Luo, L.; Zhao, S. Dynamic surface dilational properties of anionic Gemini surfactants with polyoxyethylene spacers. Colloids Surf. A 490, 41-48(2016).

44) Nash, J.J.; Erk, K.A. Stability and interfacial viscoelasticity of oil-water nanoemulsions stabilized by soy lecithin and Tween 20 for the encapsulation of bioactive carvacrol. Colloids Surf. A 517, 1-11 (2017).

45) Njauw, C.-W.; Cheng, C.-Y.; Ivanov, V.A.; Khokhlov, A.R.; Tung, S.-H. Molecular interactions between lecithin and bile salts/acids in oils and their effects on reverse micellization. Langmuir 29, 3879-3888(2013).

46) Chu, B.-S.; Rich, G.T.; Ridout, M.J.; Faulks, R.M.; Wickham, M.S.; Wilde, P.J. Modulating pancreatic lipase activity with galactolipids: effects of emulsion interfacial composition. Langmuir 25, 9352-9360 (2009).

47) Wang, C.; Chen, Z.; Dong, J.; Ullah, S.; Zhao, L.; Zhang, $\mathrm{G}$.; $\mathrm{Xu}, \mathrm{B}$. Interfacial rheological behaviors of amphiphilic sodium cholesteryl glycylglycine. Soft Matter 15, 699-708(2019).

48) Elmanan, M.; Al-Assaf, S.; Phillips, G.O.; Williams, P.A. Studies on Acacia exudate gums: Part VI. Interfacial rheology of Acacia senegal and Acacia seyal. Food Hydrocoll. 22, 682-689(2008).
49) Zhu, Y.-W.; Zhang, L.; Song, X.-W.; Luo, L.; Zhang, L.; Zhao, S.; Yu, J.-Y. Effect of electrolyte on interfacial dilational properties of chemical flooding systems by relaxation measurements. Fuel 90, 3172-3178(2011).

50) Bos, M.A.; Vlieta, T.v. Interfacial rheological properties of adsorbed protein layers and surfactants: A review. Adv. Colloid Interface Sci. 91, 437-471 (2001).

51) Tihonov, M.M.; Milyaeva, O.Y.; Noskov, B.A. Dynamic surface properties of lysozyme solutions. Impact of urea and guanidine hydrochloride. Colloid Surf. B 129, 114-120 (2015).

52) Wang, C.; Cao, X.-L.; Quo, L.-L.; Xu, Z.-C.; Zhang, L.; Gong, Q.-T.; Zhang, L.; Zhao, S. Effect of molecular structure of catanionic surfactant mixtures on their interfacial properties. Colloids Surf. A 509, 601-612 (2016).

53) Wang, C.; Fang, H.; Gong, Q.; Xu, Z.; Liu, Z.; Zhang, L.; Zhang, L.; Zhao, S. Roles of catanionic surfactant mixtures on the stability of foams in the presence of oil. Energy Fuels 30, 6355-6364(2016).

54) Wang, C.; Zhang, P.; Chen, Z.; Liu, Y.; Zhao, L.; Wang, N.; $\mathrm{Xu}, \mathrm{B}$. Effects of fatty acyl chains on the interfacial rheological behaviors of amino acid surfactants. $J$. Mol. Liq. 325, 114823(2021).

55) Chen, Z.; Zhang, P.; Liu, Y.; Mekkaoui, A.; Wang, C.; Zhao, L.; Xu, B. Interfacial rheological behavior of Nacyl amino acid surfactants derived from vegetable oils. Colloids Surf. A 592, 124600 (2020). 